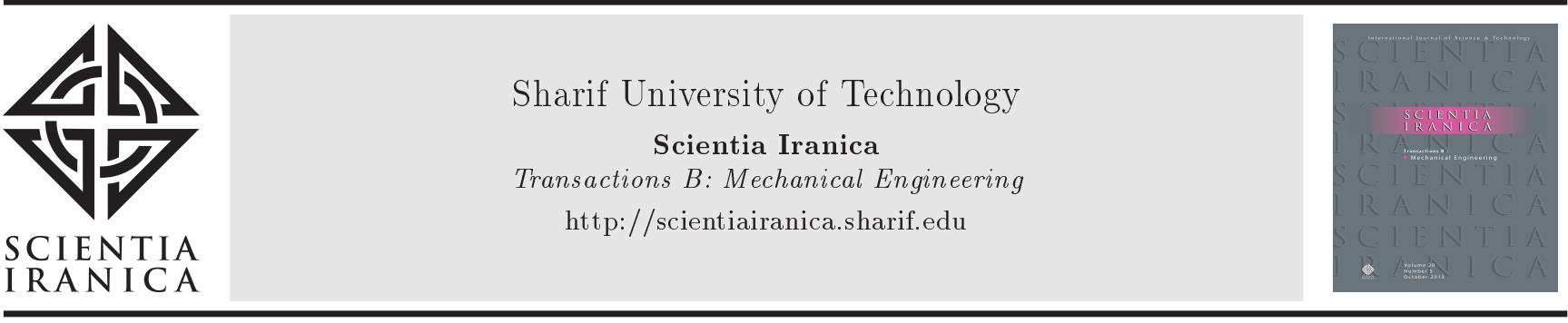

\title{
Vibration analysis of FGM rings using a newly designed cylindrical superelement
}

\author{
A.R. Fatan and M.T. Ahmadian* \\ Center of Excellence in Design, Robotics and Automation (CEDRA), School of Mechanical Engineering, Sharif University of \\ Technology, Azadi Ave, Tehran, Iran.
}

Received 26 January 2016; received in revised form 3 January 2017; accepted 20 May 2017

\author{
KEYWORDS \\ Vibration analysis; \\ Ring; \\ FGM; \\ Cylindrical \\ superelement; \\ FEM.
}

\begin{abstract}
Rings are widely used in mechanical equipment, and their fitness may undergo some damage under severe vibration. In these structures, functionally graded rings can be used to optimize the resistance, energy consumption, and fitness. Due to their complexity, the finite-element analysis may be implemented using special elements. Enhancement of accuracy and minimization of time consumption play an important role in the analysis of these rings. In this study, a new cylindrical superelement for the FGM rings is designed and implemented to facilitate the vibration analysis of the rings. The power-law distribution is used for the modeling of the FGM rings in the thickness direction. Natural frequencies and mode shapes are obtained, and results are compared with simple cases obtained from analytical solution and conventional elements. Findings indicate that, considering a few newly designed superelements, comparable results for simple cases in the reported literature can be achieved.
\end{abstract}

(C) 2018 Sharif University of Technology. All rights reserved.

\section{Introduction}

New advances in materials science have led to the creation of new materials with interesting properties. One of these materials is Functionally Graded Material (FGM). FGM is a compound that is usually made up of two components, mixing continuously. The continuous mixing causes continuous variation in material properties from one side to another. Continuity in material properties can ultimately remove the problems associated with boundaries in the laminated composite. Many researches on rings, cylinders, and FGM structures were carried out [1]. Likun Wang et al. [2] investigated the vibration of piezoceramic rings

*. Corresponding author. Tel: +982166165503 E-mail address: Ahmadian@sharif.edu (M.T. Ahmadian)

doi: $10.24200 /$ sci. 2017.4338 using theoretical analysis, numerical simulation, and experimental measurement. Mustapha [3] presented a microstructure-dependent continuum model of a microring with functionally graded material composition in the thickness direction. Loy et al. [4] used the RayleighRitz method and Love's shell theory to obtain the natural frequencies of simply supported FGM cylindrical shells. Shakeri et al. [5] presented the analysis of thick hollow cylinder made of FGM under dynamic loading. In this analysis, they assumed that FGM cylinder is made of several isotropic layers. Asgari and Akhlaghi [6] studied the natural frequency analysis of the 2D-FGM thick hollow cylinder based on threedimensional elasticity equations. Yin et al. [7] investigated free vibration analysis of functionally graded plates using isogeometric finite-element method. $\mathrm{Yu}$ et al. [8] presented geometrically nonlinear analysis of homogeneous and non-homogeneous functionally graded plates based on isogeometric analysis (IGA). 
In general, analytical methods have their own limitations and are only able to solve some specific problems. Therefore, numerical methods are used for a wide range of problems, specifically the system with nonlinear behavior. One of the most common and efficient numerical methods for solving complex problems with no analytical solution is the FiniteElement Method (FEM). In this method, the number of elements is increased to obtain convergence and improve accuracy of results. This work leads to an increase in time and memory required for solving problems. One of the attempts at improving the efficiency of the nonlinear problem simulation is the implementation of superelements. Superelements have specific shapes and features. They simplify the process of modeling for particular structures. Due to the shape of these elements, structures can be modelled with fewer elements and high accuracy. Sarvi and Ahmadian [9] designed and implemented a new spherical superelement used for static and dynamic problems. Jiang and Olson [10] extended a superelement to the nonlinear dynamic and static analyses of cylindrical shells. Koko and Olson [11] studied vibration analysis of stiffened plates using a rectangular superelement. $\mathrm{Ju}$ and Choo [12] applied the superelement approach to a cable passing through multiple pulleys. Ahmadian and Bonakdar [13] presented a new cylindrical superelement formulation and its application to structural analysis of laminated hollow cylinders. Taghvaeipour et al. [14] used this superelement for the structural analysis of functionally graded hollow cylinders. In another effort by Ghorbani and Ahmadian [15], the cylindrical superelement is developed and incorporated continuity into slope to increase the accuracy of results.

Cylindrical superelement in [15] is applied in this paper. We cannot obtain many natural frequencies and mode shapes in rings using a 16-node cylindrical superelement. Therefore, in the current work, we extend the number of nodes in [15] to 64 nodes. Then, we illustrate the shape functions, the procedure of extracting the mass, and element stiffness matrices. Homogeneous rings are analyzed, and results are compared with analytical solution and conventional elements in Abaqus. FGM rings are modelled based on varying properties in the radial direction, and the results are validated with conventional elements in Abaqus.

\section{Element definition}

Herein, a 16-node cylindrical superelement in $[13,15]$ is introduced and extended to 64 nodes. A cylindrical element with length $2 L$, inner radius $r_{1}$, and outer radius $r_{2}$ is shown in Figure 1. As shown, there are

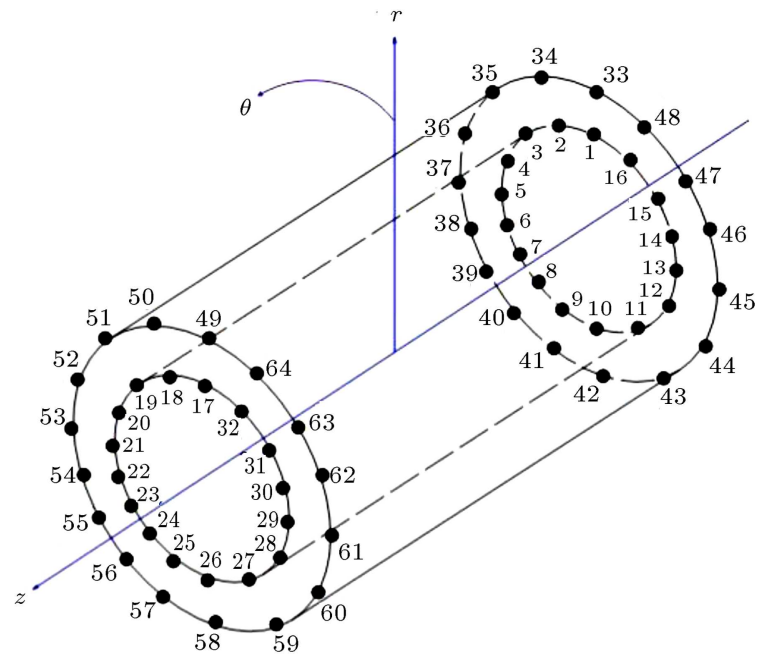

Figure 1. Superelement configuration and coordinate system.

64 nodes in each element distributed equally on both sides.

The triple cylindrical coordinate $(r-\theta-z)$ is used to determine the position vector in the element, where $r, \theta$, and $z$ are radial, tangential, and axial coordinates, respectively. The element intrinsic coordinates are defined as follows [14]:

$$
\left\{\begin{array}{l}
\eta=\frac{2 r-b}{a} \\
\gamma=\frac{\theta}{\pi}-1 \\
\xi=\frac{z}{L}
\end{array}\right.
$$

where:

$$
a=r_{2}-r_{1}, \quad b=r_{2}+r_{1} .
$$

Considering the limits for global coordinate $(r-\theta-z)$ :

$$
\left\{\begin{array}{l}
r_{1} \leq r \leq r_{2} \\
0 \leq \theta \leq 2 \pi \\
-l \leq z \leq l
\end{array}\right.
$$

implies:

$$
-1 \leq \eta, \xi, \gamma \leq 1
$$

The following conditions are essential for the shape functions of the cylindrical superelement [13].

The shape functions must be continuous and differentiable.

The shape function corresponding to each node must be one at that node and zero at other nodes.

Due to the cylindrical shape of the element, all the shape functions must be periodic with respect to tangential coordinate, $\theta$, with a period of $2 \pi$.

Satisfying the above-mentioned conditions, the newly developed shape functions are as follows: 


$$
\begin{aligned}
& N_{01}^{1}=\frac{1}{64} \cos (4 \pi \gamma)(1+\cos (4 \pi \gamma))(1+\cos (2 \pi \gamma)) \\
& (1-\cos (\pi \gamma))(1-\eta)\left(2-3 \xi+\xi^{3}\right), \\
& N_{11}^{1}=\frac{1}{64} \cos (4 \pi \gamma)(1+\cos (4 \pi \gamma))(1+\cos (2 \pi \gamma)) \\
& (1-\cos (\pi \gamma))(1-\eta)\left(1-\xi-\xi^{2}+\xi^{3}\right), \\
& N_{1}=\frac{1}{32} \cos (4 \pi \gamma)(1+\cos (4 \pi \gamma))(1+\cos (2 \pi \gamma)) \\
& (1-\cos (\pi \gamma))(1-\eta)(1-\xi) \\
& N_{02}^{1}=\frac{1}{64} \sin (4 \pi \gamma)(1+\sin (4 \pi \gamma))\left(1+\sin \left(2 \pi \gamma+\frac{\pi}{4}\right)\right) \\
& \left(1-\sin \left(\pi \gamma+\frac{3 \pi}{8}\right)\right)(1-\eta)\left(2-3 \xi+\xi^{3}\right) \\
& N_{12}^{1}=\frac{1}{64} \sin (4 \pi \gamma)(1+\sin (4 \pi \gamma))\left(1+\sin \left(2 \pi \gamma+\frac{\pi}{4}\right)\right) \\
& \left(1-\sin \left(\pi \gamma+\frac{3 \pi}{8}\right)\right)(1-\eta)\left(1-\xi-\xi^{2}+\xi^{3}\right), \\
& N_{2}=\frac{1}{32} \sin (4 \pi \gamma)(1+\sin (4 \pi \gamma))\left(1+\sin \left(2 \pi \gamma+\frac{\pi}{4}\right)\right) \\
& \left(1-\sin \left(\pi \gamma+\frac{3 \pi}{8}\right)\right)(1-\eta)(1-\xi) \\
& N_{03}^{1}=-\frac{1}{64} \cos (4 \pi \gamma)(1-\cos (4 \pi \gamma))(1+\sin (2 \pi \gamma)) \\
& \left(1-\sin \left(\pi \gamma+\frac{\pi}{4}\right)\right)(1-\eta)\left(2-3 \xi+\xi^{3}\right), \\
& N_{13}^{1}=-\frac{1}{64} \cos (4 \pi \gamma)(1-\cos (4 \pi \gamma))(1+\sin (2 \pi \gamma)) \\
& \left(1-\sin \left(\pi \gamma+\frac{\pi}{4}\right)\right)(1-\eta)\left(1-\xi-\xi^{2}+\xi^{3}\right), \\
& N_{3}=-\frac{1}{32} \cos (4 \pi \gamma)(1-\cos (4 \pi \gamma))(1+\sin (2 \pi \gamma)) \\
& \left(1-\sin \left(\pi \gamma+\frac{\pi}{4}\right)\right)(1-\eta)(1-\xi) \\
& N_{04}^{1}=-\frac{1}{64} \sin (4 \pi \gamma)(1-\sin (4 \pi \gamma)) \\
& \left(1-\cos \left(2 \pi \gamma+\frac{\pi}{4}\right)\right)\left(1-\sin \left(\pi \gamma+\frac{\pi}{8}\right)\right) \\
& (1-\eta)\left(2-3 \xi+\xi^{3}\right), \\
& N_{14}^{1}=-\frac{1}{64} \sin (4 \pi \gamma)(1-\sin (4 \pi \gamma)) \\
& \left(1-\cos \left(2 \pi \gamma+\frac{\pi}{4}\right)\right)\left(1-\sin \left(\pi \gamma+\frac{\pi}{8}\right)\right) \\
& (1-\eta)\left(1-\xi-\xi^{2}+\xi^{3}\right), \\
& N_{4}=-\frac{1}{32} \sin (4 \pi \gamma)(1-\sin (4 \pi \gamma))\left(1-\cos \left(2 \pi \gamma+\frac{\pi}{4}\right)\right) \\
& \left(1-\sin \left(\pi \gamma+\frac{\pi}{8}\right)\right)(1-\eta)(1-\xi), \\
& N_{05}^{1}=\frac{1}{64} \cos (4 \pi \gamma)(1+\cos (4 \pi \gamma))(1-\cos (2 \pi \gamma)) \\
& (1-\sin (\pi \gamma))(1-\eta)\left(2-3 \xi+\xi^{3}\right), \\
& N_{15}^{1}=\frac{1}{64} \cos (4 \pi \gamma)(1+\cos (4 \pi \gamma))(1-\cos (2 \pi \gamma)) \\
& (1-\sin (\pi \gamma))(1-\eta)\left(1-\xi-\xi^{2}+\xi^{3}\right), \\
& N_{5}=\frac{1}{32} \cos (4 \pi \gamma)(1+\cos (4 \pi \gamma))(1-\cos (2 \pi \gamma)) \\
& (1-\sin (\pi \gamma))(1-\eta)(1-\xi), \\
& N_{06}^{1}=\frac{1}{64} \sin (4 \pi \gamma)(1+\sin (4 \pi \gamma))\left(1-\sin \left(2 \pi \gamma+\frac{\pi}{4}\right)\right) \\
& \left(1+\cos \left(\pi \gamma+\frac{3 \pi}{8}\right)\right)(1-\eta)\left(2-3 \xi+\xi^{3}\right), \\
& N_{16}^{1}=\frac{1}{64} \sin (4 \pi \gamma)(1+\sin (4 \pi \gamma))\left(1-\sin \left(2 \pi \gamma+\frac{\pi}{4}\right)\right) \\
& \left(1+\cos \left(\pi \gamma+\frac{3 \pi}{8}\right)\right)(1-\eta)\left(1-\xi-\xi^{2}+\xi^{3}\right), \\
& N_{6}=\frac{1}{32} \sin (4 \pi \gamma)(1+\sin (4 \pi \gamma))\left(1-\sin \left(2 \pi \gamma+\frac{\pi}{4}\right)\right) \\
& \left(1+\cos \left(\pi \gamma+\frac{3 \pi}{8}\right)\right)(1-\eta)(1-\xi) \\
& N_{07}^{1}=-\frac{1}{64} \cos (4 \pi \gamma)(1-\cos (4 \pi \gamma))(1-\sin (2 \pi \gamma)) \\
& \left(1+\cos \left(\pi \gamma+\frac{\pi}{4}\right)\right)(1-\eta)\left(2-3 \xi+\xi^{3}\right), \\
& N_{17}^{1}=-\frac{1}{64} \cos (4 \pi \gamma)(1-\cos (4 \pi \gamma))(1-\sin (2 \pi \gamma)) \\
& \left(1+\cos \left(\pi \gamma+\frac{\pi}{4}\right)\right)(1-\eta)\left(1-\xi-\xi^{2}+\xi^{3}\right), \\
& N_{7}=-\frac{1}{32} \cos (4 \pi \gamma)(1-\cos (4 \pi \gamma))(1-\sin (2 \pi \gamma)) \\
& \left(1+\cos \left(\pi \gamma+\frac{\pi}{4}\right)\right)(1-\eta)(1-\xi)
\end{aligned}
$$




$$
\begin{aligned}
N_{08}^{1}= & -\frac{1}{64} \sin (4 \pi \gamma)(1-\sin (4 \pi \gamma)) \\
& \left(1+\cos \left(2 \pi \gamma+\frac{\pi}{4}\right)\right)\left(1+\cos \left(\pi \gamma+\frac{\pi}{8}\right)\right) \\
& (1-\eta)\left(2-3 \xi+\xi^{3}\right), \\
N_{18}^{1}= & -\frac{1}{64} \sin (4 \pi \gamma)(1-\sin (4 \pi \gamma)) \\
& \left(1+\cos \left(2 \pi \gamma+\frac{\pi}{4}\right)\right)\left(1+\cos \left(\pi \gamma+\frac{\pi}{8}\right)\right) \\
& (1-\eta)\left(1-\xi-\xi^{2}+\xi^{3}\right),
\end{aligned}
$$$$
N_{8}=-\frac{1}{32} \sin (4 \pi \gamma)(1-\sin (4 \pi \gamma))\left(1+\cos \left(2 \pi \gamma+\frac{\pi}{4}\right)\right)
$$$$
\left(1+\cos \left(\pi \gamma+\frac{\pi}{8}\right)\right)(1-\eta)(1-\xi)
$$$$
N_{09}^{1}=\frac{1}{64} \cos (4 \pi \gamma)(1+\cos (4 \pi \gamma))(1+\cos (2 \pi \gamma))
$$$$
(1+\cos (\pi \gamma))(1-\eta)\left(2-3 \xi+\xi^{3}\right)
$$$$
N_{19}^{1}=\frac{1}{64} \cos (4 \pi \gamma)(1+\cos (4 \pi \gamma))(1+\cos (2 \pi \gamma))
$$$$
(1+\cos (\pi \gamma))(1-\eta)\left(1-\xi-\xi^{2}+\xi^{3}\right),
$$$$
N_{9}=\frac{1}{32} \cos (4 \pi \gamma)(1+\cos (4 \pi \gamma))(1+\cos (2 \pi \gamma))
$$$$
(1+\cos (\pi \gamma))(1-\eta)(1-\xi)
$$$$
N_{010}^{1}=\frac{1}{64} \sin (4 \pi \gamma)(1+\sin (4 \pi \gamma))\left(1+\sin \left(2 \pi \gamma+\frac{\pi}{4}\right)\right)
$$$$
\left(1+\sin \left(\pi \gamma+\frac{3 \pi}{8}\right)\right)(1-\eta)\left(2-3 \xi+\xi^{3}\right),
$$$$
N_{110}^{1}=\frac{1}{64} \sin (4 \pi \gamma)(1+\sin (4 \pi \gamma))\left(1+\sin \left(2 \pi \gamma+\frac{\pi}{4}\right)\right)
$$$$
\left(1+\sin \left(\pi \gamma+\frac{3 \pi}{8}\right)\right)(1-\eta)\left(1-\xi-\xi^{2}+\xi^{3}\right),
$$$$
N_{10}=\frac{1}{32} \sin (4 \pi \gamma)(1+\sin (4 \pi \gamma))\left(1+\sin \left(2 \pi \gamma+\frac{\pi}{4}\right)\right)
$$$$
\left(1+\sin \left(\pi \gamma+\frac{3 \pi}{8}\right)\right)(1-\eta)(1-\xi),
$$$$
N_{011}^{1}=-\frac{1}{64} \cos (4 \pi \gamma)(1-\cos (4 \pi \gamma))(1+\sin (2 \pi \gamma))
$$

$$
\left(1+\sin \left(\pi \gamma+\frac{\pi}{4}\right)\right)(1-\eta)\left(2-3 \xi+\xi^{3}\right)
$$

$$
\begin{aligned}
N_{111}^{1}= & -\frac{1}{64} \cos (4 \pi \gamma)(1-\cos (4 \pi \gamma))(1+\sin (2 \pi \gamma)) \\
& \left(1+\sin \left(\pi \gamma+\frac{\pi}{4}\right)\right)(1-\eta)\left(1-\xi-\xi^{2}+\xi^{3}\right),
\end{aligned}
$$

$$
N_{11}=-\frac{1}{32} \cos (4 \pi \gamma)(1-\cos (4 \pi \gamma))(1+\sin (2 \pi \gamma))
$$$$
\left(1+\sin \left(\pi \gamma+\frac{\pi}{4}\right)\right)(1-\eta)(1-\xi)
$$$$
N_{012}^{1}=-\frac{1}{64} \sin (4 \pi \gamma)(1-\sin (4 \pi \gamma))\left(1-\cos \left(2 \pi \gamma+\frac{\pi}{4}\right)\right)
$$$$
\left(1+\sin \left(\pi \gamma+\frac{\pi}{8}\right)\right)(1-\eta)\left(2-3 \xi+\xi^{3}\right)
$$$$
N_{112}^{1}=-\frac{1}{64} \sin (4 \pi \gamma)(1-\sin (4 \pi \gamma))\left(1-\cos \left(2 \pi \gamma+\frac{\pi}{4}\right)\right)
$$$$
\left(1+\sin \left(\pi \gamma+\frac{\pi}{8}\right)\right)(1-\eta)\left(1-\xi-\xi^{2}+\xi^{3}\right)
$$

$$
\begin{aligned}
N_{12}= & -\frac{1}{32} \sin (4 \pi \gamma)(1-\sin (4 \pi \gamma))\left(1-\cos \left(2 \pi \gamma+\frac{\pi}{4}\right)\right) \\
& \left(1+\sin \left(\pi \gamma+\frac{\pi}{8}\right)\right)(1-\eta)(1-\xi),
\end{aligned}
$$$$
N_{013}^{1}=\frac{1}{64} \cos (4 \pi \gamma)(1+\cos (4 \pi \gamma))(1-\cos (2 \pi \gamma))
$$$$
(1+\sin (\pi \gamma))(1-\eta)\left(2-3 \xi+\xi^{3}\right),
$$$$
N_{113}^{1}=\frac{1}{64} \cos (4 \pi \gamma)(1+\cos (4 \pi \gamma))(1-\cos (2 \pi \gamma))
$$

$$
(1+\sin (\pi \gamma))(1-\eta)\left(1-\xi-\xi^{2}+\xi^{3}\right),
$$

$$
N_{13}=\frac{1}{32} \cos (4 \pi \gamma)(1+\cos (4 \pi \gamma))(1-\cos (2 \pi \gamma))
$$$$
(1+\sin (\pi \gamma))(1-\eta)(1-\xi)
$$

$$
N_{014}^{1}=\frac{1}{64} \sin (4 \pi \gamma)(1+\sin (4 \pi \gamma))\left(1-\sin \left(2 \pi \gamma+\frac{\pi}{4}\right)\right)
$$$$
\left(1-\cos \left(\pi \gamma+\frac{3 \pi}{8}\right)\right)(1-\eta)\left(2-3 \xi+\xi^{3}\right),
$$

$$
\begin{aligned}
N_{114}^{1}= & \frac{1}{64} \sin (4 \pi \gamma)(1+\sin (4 \pi \gamma))\left(1-\sin \left(2 \pi \gamma+\frac{\pi}{4}\right)\right) \\
& \left(1-\cos \left(\pi \gamma+\frac{3 \pi}{8}\right)\right)(1-\eta)\left(1-\xi-\xi^{2}+\xi^{3}\right),
\end{aligned}
$$




$$
\begin{aligned}
N_{14}= & \frac{1}{32} \sin (4 \pi \gamma)(1+\sin (4 \pi \gamma))\left(1-\sin \left(2 \pi \gamma+\frac{\pi}{4}\right)\right) \\
& \left(1-\cos \left(\pi \gamma+\frac{3 \pi}{8}\right)\right)(1-\eta)(1-\xi), \\
N_{015}^{1}= & -\frac{1}{64} \cos (4 \pi \gamma)(1-\cos (4 \pi \gamma))(1-\sin (2 \pi \gamma)) \\
& \left(1-\cos \left(\pi \gamma+\frac{\pi}{4}\right)\right)(1-\eta)\left(2-3 \xi+\xi^{3}\right), \\
N_{115}^{1}= & -\frac{1}{64} \cos (4 \pi \gamma)(1-\cos (4 \pi \gamma))(1-\sin (2 \pi \gamma)) \\
& \left(1-\cos \left(\pi \gamma+\frac{\pi}{4}\right)\right)(1-\eta)\left(1-\xi-\xi^{2}+\xi^{3}\right), \\
N_{15}= & \frac{1}{32} \cos (4 \pi \gamma)(1-\cos (4 \pi \gamma))(1-\sin (2 \pi \gamma)) \\
& \left(1-\cos \left(\pi \gamma+\frac{\pi}{4}\right)\right)(1-\eta)(1-\xi),
\end{aligned}
$$$$
N_{016}^{1}=-\frac{1}{64} \sin (4 \pi \gamma)(1-\sin (4 \pi \gamma))\left(1+\cos \left(2 \pi \gamma+\frac{\pi}{4}\right)\right)
$$$$
\left(1-\cos \left(\pi \gamma+\frac{\pi}{8}\right)\right)(1-\eta)\left(2-3 \xi+\xi^{3}\right)
$$$$
N_{116}^{1}=-\frac{1}{64} \sin (4 \pi \gamma)(1-\sin (4 \pi \gamma))
$$$$
\left(1+\cos \left(2 \pi \gamma+\frac{\pi}{4}\right)\right)\left(1-\cos \left(\pi \gamma+\frac{\pi}{8}\right)\right)
$$$$
(1-\eta)\left(1-\xi-\xi^{2}+\xi^{3}\right),
$$$$
N_{16}=-\frac{1}{32} \sin (4 \pi \gamma)(1-\sin (4 \pi \gamma))\left(1+\cos \left(2 \pi \gamma+\frac{\pi}{4}\right)\right)
$$$$
\left(1-\cos \left(\pi \gamma+\frac{\pi}{8}\right)\right)(1-\eta)(1-\xi)
$$$$
N_{017}^{1}=\frac{1}{64} \cos (4 \pi \gamma)(1+\cos (4 \pi \gamma))(1+\cos (2 \pi \gamma))
$$$$
(1-\cos (\pi \gamma))(1-\eta)\left(2+3 \xi-\xi^{3}\right),
$$$$
N_{117}^{1}=\frac{1}{64} \cos (4 \pi \gamma)(1+\cos (4 \pi \gamma))(1+\cos (2 \pi \gamma))
$$$$
(1-\cos (\pi \gamma))(1-\eta)\left(-1-\xi+\xi^{2}+\xi^{3}\right),
$$$$
N_{17}=\frac{1}{32} \cos (4 \pi \gamma)(1+\cos (4 \pi \gamma))(1+\cos (2 \pi \gamma))
$$$$
(1-\cos (\pi \gamma))(1-\eta)(1+\xi),
$$

$$
\begin{aligned}
& N_{032}^{1}=-\frac{1}{64} \sin (4 \pi \gamma)(1-\sin (4 \pi \gamma)) \\
& \left(1+\cos \left(2 \pi \gamma+\frac{\pi}{4}\right)\right)\left(1-\cos \left(\pi \gamma+\frac{\pi}{8}\right)\right) \\
& (1-\eta)\left(2+3 \xi-\xi^{3}\right) \\
& N_{132}^{1}=-\frac{1}{64} \sin (4 \pi \gamma)(1-\sin (4 \pi \gamma))\left(1+\cos \left(2 \pi \gamma+\frac{\pi}{4}\right)\right) \\
& \left(1-\cos \left(\pi \gamma+\frac{\pi}{8}\right)\right)(1-\eta)\left(-1-\xi+\xi^{2}+\xi^{3}\right), \\
& N_{32}=-\frac{1}{32} \sin (4 \pi \gamma)(1-\sin (4 \pi \gamma))\left(1+\cos \left(2 \pi \gamma+\frac{\pi}{4}\right)\right) \\
& \left(1-\cos \left(\pi \gamma+\frac{\pi}{8}\right)\right)(1-\eta)(1+\xi), \\
& N_{033}^{1}=\frac{1}{64} \cos (4 \pi \gamma)(1+\cos (4 \pi \gamma))(1+\cos (2 \pi \gamma)) \\
& (1-\cos (\pi \gamma))(1+\eta)\left(2-3 \xi+\xi^{3}\right), \\
& N_{133}^{1}=\frac{1}{64} \cos (4 \pi \gamma)(1+\cos (4 \pi \gamma))(1+\cos (2 \pi \gamma)) \\
& (1-\cos (\pi \gamma))(1+\eta)\left(1-\xi-\xi^{2}+\xi^{3}\right), \\
& N_{33}=\frac{1}{32} \cos (4 \pi \gamma)(1+\cos (4 \pi \gamma))(1+\cos (2 \pi \gamma)) \\
& (1-\cos (\pi \gamma))(1+\eta)(1-\xi), \\
& N_{064}^{1}=-\frac{1}{64} \sin (4 \pi \gamma)(1-\sin (4 \pi \gamma))\left(1+\cos \left(2 \pi \gamma+\frac{\pi}{4}\right)\right) \\
& \left(1-\cos \left(\pi \gamma+\frac{\pi}{8}\right)\right)(1+\eta)\left(2+3 \xi-\xi^{3}\right), \\
& N_{164}^{1}=-\frac{1}{64} \sin (4 \pi \gamma)(1-\sin (4 \pi \gamma)) \\
& \left(1+\cos \left(2 \pi \gamma+\frac{\pi}{4}\right)\right)\left(1-\cos \left(\pi \gamma+\frac{\pi}{8}\right)\right) \\
& (1+\eta)\left(-1-\xi+\xi^{2}+\xi^{3}\right), \\
& N_{64}=-\frac{1}{32} \sin (4 \pi \gamma)(1-\sin (4 \pi \gamma)) \\
& \left(1+\cos \left(2 \pi \gamma+\frac{\pi}{4}\right)\right)\left(1-\cos \left(\pi \gamma+\frac{\pi}{8}\right)\right) \\
& (1+\eta)(1+\xi)(5) .
\end{aligned}
$$

According to Eq. (5), the shape function corresponding to each node gets the value of one at that node and 
zero at other nodes. For example, the first node with intrinsic coordinates $\eta=-1, \xi=-1$, and $\gamma=-1$ is one in the first shape function and zero in the others.

\section{Stress-strain relations}

The displacement vector in this element is [13]:

$$
u=\left[\begin{array}{lll}
u_{r} & u_{\theta} & u_{z}
\end{array}\right]^{T}
$$

$u$ is obtained using nodal displacement vector, $q$, and the shape functions according to:

$$
u=N q
$$

$q$ and $N$ can be obtained by Eqs. (8) and (9) as shown in Box I.

Strain vector in cylindrical coordinate system is defined as follows:

$$
\varepsilon=\left[\begin{array}{llllll}
\varepsilon_{r} & \varepsilon_{\theta} & \varepsilon_{z} & \gamma_{r \theta} & \gamma_{r z} & \gamma_{\theta z}
\end{array}\right]^{T} .
$$

The strain-displacement relations can be stated in a matrix form in the cylindrical coordinate as follows [16]

$$
\varepsilon=L u,
$$

where $L$ is the operator matrix as follows:

$$
L=\left[\begin{array}{ccc}
\frac{\partial}{\partial r} & 0 & 0 \\
\frac{1}{r} & \frac{1}{r} \frac{\partial}{\partial \theta} & 0 \\
0 & 0 & \frac{\partial}{\partial z} \\
\frac{1}{r} \frac{\partial}{\partial \theta} & \frac{\partial}{\partial r}-\frac{1}{r} & 0 \\
\frac{\partial}{\partial z} & 0 & \frac{\partial}{\partial r} \\
0 & \frac{\partial}{\partial z} & \frac{1}{r} \frac{\partial}{\partial \theta}
\end{array}\right]
$$

By considering Eqs. (7) and (11), the strain vector could be written as follows:

$$
\varepsilon=B q,
$$

where $B$ is obtained from:

$$
B=L N \text {. }
$$

In cylindrical coordinate system, the stress vector is defined as follows:

$$
\sigma=\left[\begin{array}{llllll}
\sigma_{r} & \sigma_{\theta} & \sigma_{z} & \tau_{r \theta} & \tau_{r z} & \tau_{\theta z}
\end{array}\right]^{T},
$$

which is related to the strain vector according to the following equation [16]:

$$
\sigma=D \varepsilon
$$

where $D$ is the property matrix given by:

$$
\begin{aligned}
& D=\frac{E}{1+\nu}\left[\begin{array}{llllll}
d & e & e & 0 & 0 & 0 \\
e & d & e & 0 & 0 & 0 \\
e & e & d & 0 & 0 & 0 \\
0 & 0 & 0 & \frac{1}{2} & 0 & 0 \\
0 & 0 & 0 & 0 & \frac{1}{2} & 0 \\
0 & 0 & 0 & 0 & 0 & \frac{1}{2}
\end{array}\right] \\
& e=\frac{\nu}{1-2 \nu} \\
& d=\frac{1-\nu}{1-2 \nu}
\end{aligned}
$$

where $\nu$ and $E$ are Poisson's ratio and modulus of elasticity, respectively.

\section{Element stiffness and mass matrices}

Element stiffness and mass matrices should be integrated on the volume of element. In cylindrical coordinate $(r-\theta-z)$, infinitesimal volume, $d \nu$, can be expressed as follows:

$$
d \nu_{\text {global }}=r d r d \theta d z \text {. }
$$

In the local coordinate, infinitesimal volume, $d \nu$, can be expressed as follows:

$$
d \nu_{\text {local }}=\frac{1}{2} \operatorname{det}(J)(a \eta+b) d \eta d \gamma d \xi,
$$

where $J$ is the Jacobian matrix and is calculated as follows:

$$
J=\left[\begin{array}{lll}
\frac{\partial r}{\partial \eta} & \frac{\partial \theta}{\partial \eta} & \frac{\partial z}{\partial \eta} \\
\frac{\partial r}{\partial \gamma} & \frac{\partial \theta}{\partial \gamma} & \frac{\partial z}{\partial \gamma} \\
\frac{\partial r}{\partial \xi} & \frac{\partial \theta}{\partial \xi} & \frac{\partial z}{\partial \xi}
\end{array}\right]=\left[\begin{array}{ccc}
\frac{a}{2} & 0 & 0 \\
0 & \pi & 0 \\
0 & 0 & l
\end{array}\right]
$$

$$
\begin{aligned}
q & =\left[u_{1 r} \frac{\partial u_{1 r}}{\partial z} u_{1 \theta} \frac{\partial u_{1 \theta}}{\partial z} u_{1 z} \ldots u_{64 r} \frac{\partial u_{64 r}}{\partial z} u_{64 \theta} \frac{\partial u_{64 \theta}}{\partial z} u_{64 z}\right]^{T}, \\
N & =\left[\begin{array}{ccccccccccc}
N^{1} 01 & N^{1}{ }_{11} & 0 & 0 & 0 & \cdots & N^{1} 064 & N^{1}{ }^{164} & 0 & 0 & 0 \\
0 & 0 & N^{1}{ }_{01} & N^{1}{ }_{11} & 0 & \cdots & 0 & 0 & N^{1} 064 & N^{1} 164 & 0 \\
0 & 0 & 0 & 0 & N_{1} & \cdots & 0 & 0 & 0 & 0 & N_{64}
\end{array}\right],
\end{aligned}
$$

in which $N_{i}$ represents the shape functions. 
which has the determinant:

$$
\operatorname{det}(J)=\frac{\pi a l}{2}
$$

The element stiffness and mass matrices are defined as follows [17]:

$$
\begin{aligned}
& K^{e}=\int_{V^{e}} B^{T} D B d \nu, \\
& M^{e}=\int_{V^{e}} N^{T} \rho N d \nu .
\end{aligned}
$$

Using Eqs. (19) and (21), we get:

$$
\begin{aligned}
K^{e} & =\int_{-1}^{1} \int_{-1}^{1} \int_{-1}^{1} B^{T} D B \frac{l a \pi}{4}(a \eta+b) d \eta d \xi d \gamma, \\
M^{e} & =\int_{-1}^{1} \int_{-1}^{1} \int_{-1}^{1} N^{T} \rho N \frac{l a \pi}{4}(a \eta+b) d \eta d \xi d \gamma .
\end{aligned}
$$

\section{Modeling of FGM}

Properties of the functionally graded materials change smoothly with their position. For FG rings considered in this work, the properties are only a function of radial position and stay constant relative to longitudinal and angular directions. Many relations are available to describe the changing properties of functionally graded materials, and the following relation is assumed in this study [14]:

$$
P(r)=\left(P_{m}-P_{c}\right)\left(\frac{r-r_{i}}{r_{o}-r_{i}}\right)^{n}+P_{c}
$$

where $P(r)$ is the property of FGM which can be physical properties such as modulus of elasticity, Poisson's ratio, thermal conductivity, density, etc. $P_{c}$ (ceramic) and $P_{m}$ (metal) are the properties of the inner and outer parts of the ring, respectively. $n$ is gradient index, and $r_{i}$ and $r_{o}$ are inner and outer radii, respectively. Through substituting modulus of elasticity, Poisson's ratio, and density of materials into Eq. (26) and using Eqs. (17), (24), and (25), the FG ring can be modeled.

\section{Modal analysis}

The equation of motion for an undamped multi degree of freedom system is defined as follows [14]:

$$
M \ddot{q}+K q=0,
$$

where $q$ is the global degree of freedom vector, and $K$ and $M$ are stiffness and mass matrices, respectively.
Vibrating the system in one of the mode shapes, $\varphi_{i}$, leads to a simple harmonic motion with the corresponding natural frequency, $\omega_{i}$, which could be expressed as:

$$
q=Q_{i} \sin \left(\omega_{i} t\right)
$$

where $Q_{i}$ is the amplitude vector. Substituting Eq. (28) into Eq. (27) yields:

$$
\left(-M \omega_{i}^{2}+K\right) Q_{i}=0 .
$$

To find a nontrivial solution to the above equation, the determinant of the coefficient matrix should be 0 :

$$
\left|-M \omega_{i}^{2}+K\right|=0 .
$$

Premultiplying the equation by $M^{-1}$ and defining $D=$ $M^{-1} \times K, \lambda_{i}=\omega_{i}^{2}$ results in:

$$
\left|D-I \lambda_{i}\right|=0 \text {. }
$$

Solving the eigenvalue problem in Eq. (31) yields eigenvalues and eigenvectors of $D$, which are the square of natural frequencies and the mode and shapes of the system, respectively.

\section{Example}

Consider a FGM free ring with thickness of $L=$ $0.01 \mathrm{~m}$, inner radius $=0.05 \mathrm{~m}$, outer radius $=0.052 \mathrm{~m}$, inner material $=\mathrm{Si}_{3} \mathrm{~N}_{4}$, and outer material $=$ stainless steel (SUS304) whose material properties are given in Table 1.

The ring is modelled with 6 cylindrical superelements in the radial direction according to the result of Figure 5. Initially, let the ring be homogeneous, only ceramic or only metal $(n=\infty, 0$ in Eq. (26)). The first three natural frequencies obtained using superelement for in-plane flexural vibrations of ring are compared with those obtained from Abaqus and analytical solution in ref [1] in Tables 2 and 3 .

The first three mode shapes of the ring are shown in Figures 2 to 4.

Assume that the properties vary from the inner to outer surfaces linearly $(n=1)$. The first three natural frequencies obtained from superelement are compared with those obtained from Abaqus in Table 4.

To investigate the convergence of the results, number of cylindrical superelement in the radial direction is increased for the case of $n=1$. Findings

Table 1. Material properties of FGM.

\begin{tabular}{ccc}
\hline Properties & $\mathbf{S i}_{\mathbf{3}} \mathbf{N}_{\mathbf{4}}$ & Stainless steel (SUS304) \\
\hline$E(\mathrm{GPa})$ & 315.7 & 207.8 \\
$\nu$ & 0.24 & 0.318 \\
$\left.\rho \mathrm{kg} / \mathrm{m}^{3}\right)$ & 2370 & 8166 \\
\hline
\end{tabular}


Table 2. The first three natural frequencies for $n=0$ (SUS304).

\begin{tabular}{lcccccc}
\hline & \multicolumn{2}{c}{ Method } & & \multicolumn{2}{c}{ Relative error $^{\mathbf{b}}$} \\
\cline { 2 - 3 } & Superelement (6) & Abaqus (10000) & Ref. $[\mathbf{1}]$ & & Superelement & Abaqus \\
\hline The first natural frequency $(\mathrm{Hz})$ & 479 & 474 & 478 & & $0.21 \%$ & $0.84 \%$ \\
The second natural frequency $(\mathrm{Hz})$ & 1355 & 1340 & 1353 & & $0.15 \%$ & $0.96 \%$ \\
The third natural frequency $(\mathrm{Hz})$ & 2596 & 2566 & 2593 & & $0.12 \%$ & $1.04 \%$ \\
\hline
\end{tabular}

a The number indicates the number of elements; ${ }^{b}$ With respect to the analytical method [1].

Table 3. The first three natural frequencies for $n=\infty\left(\mathrm{Si}_{3} \mathrm{~N}_{4}\right)$.

\begin{tabular}{lccccccc}
\hline & \multicolumn{3}{c}{ Method } & & \multicolumn{2}{c}{ Relative error $^{\mathbf{b}}$} \\
\cline { 2 - 3 } & & Superelement (6) & Abaqus (10000) & Ref. [1] & & Superelement & Abaqus \\
\hline The first natural frequency $(\mathrm{Hz})$ & 1095 & 1082 & 1094 & & $0.09 \%$ & $1.1 \%$ \\
The second natural frequency $(\mathrm{Hz})$ & 3094 & 3052 & 3095 & & $0.03 \%$ & $1.39 \%$ \\
The third natural frequency $(\mathrm{Hz})$ & 5928 & 5846 & 5934 & & $0.1 \%$ & $1.48 \%$ \\
\hline
\end{tabular}

a The number indicates the number of elements; $b$ With respect to the analytical method [1].

indicate that six superelements are sufficient for relatively accurate result. Figure 5 presents convergence of the first natural frequency for different number of layers.

It is clear that using six superelements results in

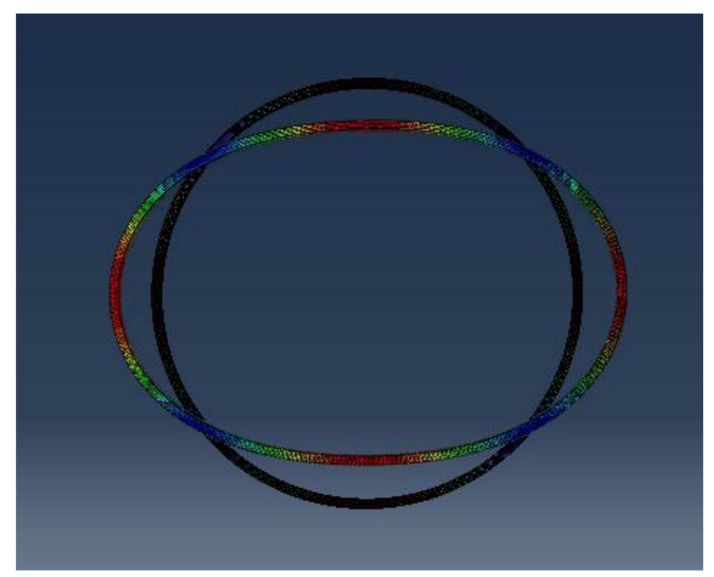

Figure 2. The first mode shape.

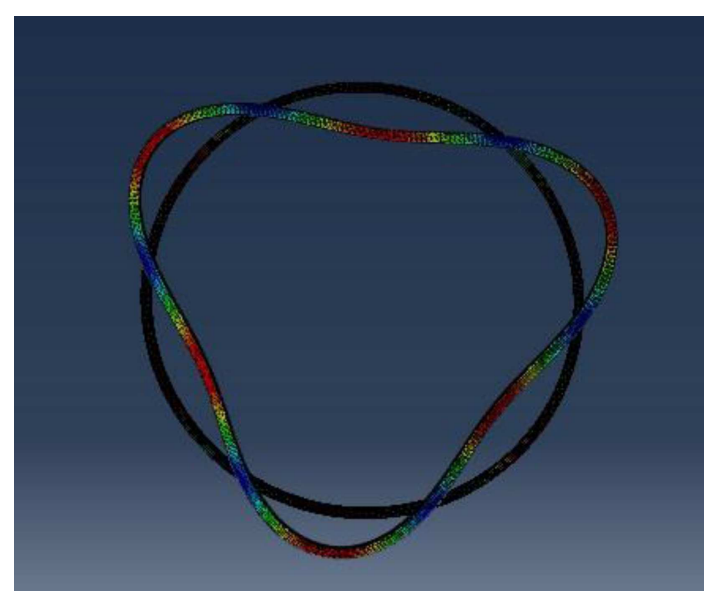

Figure 3. The second mode shape. good convergence with $0.2 \%$ accuracy. The effect of gradient index $n$ on natural frequencies is presented in Figures 6-8.

It can be observed that natural frequency increases as $n$ increases due to higher volume fraction of ceramic with larger stiffness and lower density with respect to metal.

Figures 9-11 depict the first three natural frequencies at different values of the ratio of thickness to radius $\left(h / r_{i}\right)$ for different $n$.

It is clear that as the ratio of thickness to radius increases, the natural frequency also increases.

Natural frequencies of rings obtained using superelement, Abaqus software, and analytical solution for $n=0$ (metal), $n=\infty$ (ceramic), and $n=1$ (FGM) are presented in Tables 2-4. Findings indicate that it is highly possible to predict the results within $0.21 \%$ error with the application of six superelements, while the predicted error will be $1.48 \%$ with respect to analytical solution using 10000 elements through Abaqus software.

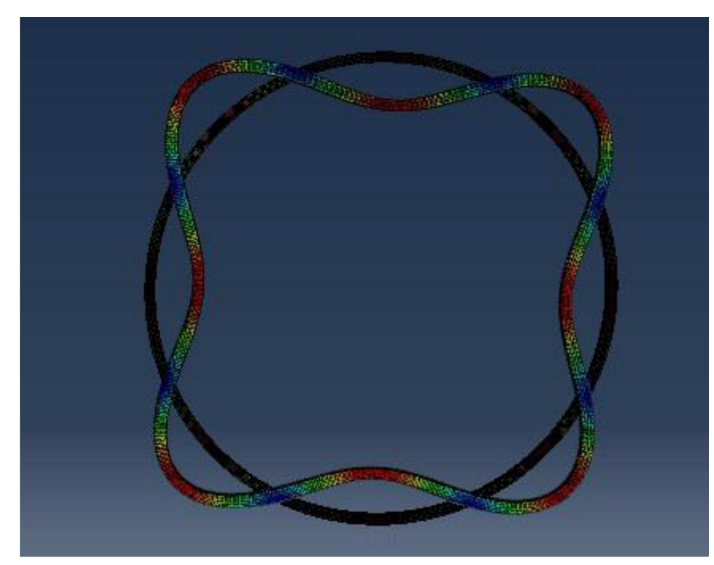

Figure 4. The third mode shape. 
Table 4. The first three natural frequencies for $n=1$.

\begin{tabular}{lccc}
\hline & \multicolumn{2}{c}{ Method } & \\
\cline { 2 - 3 } & Superelement & Abaqus & Relative error ${ }^{\mathrm{a}}$ \\
\hline The first natural frequency $(\mathrm{Hz})$ & 666 & 661 & $0.76 \%$ \\
The second natural frequency $(\mathrm{Hz})$ & 1884 & 1871 & $0.69 \%$ \\
The third natural frequency $(\mathrm{Hz})$ & 3611 & 3585 & $0.73 \%$ \\
\hline
\end{tabular}

${ }^{\text {a }}$ With respect to Abaqus

\section{Conclusion}

In this paper, a 64-node cylindrical superelement is developed, and vibration analysis of functionally graded rings using newly designed cylindrical superelement is

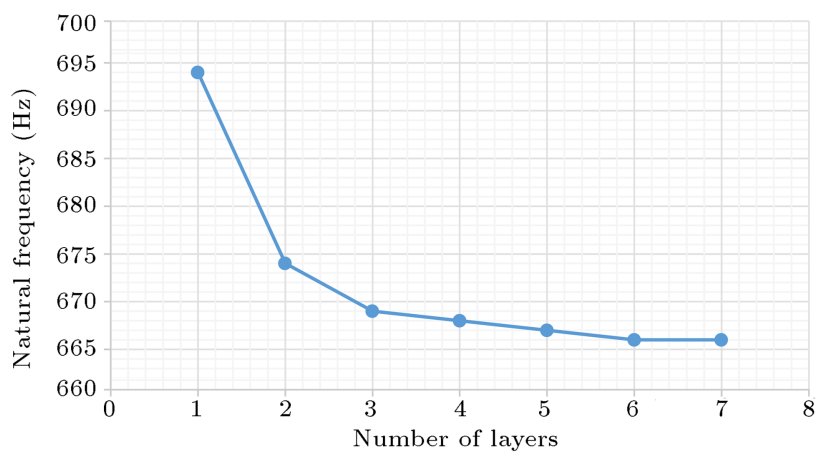

Figure 5. The first natural frequency for different number of layers and $n=1$.

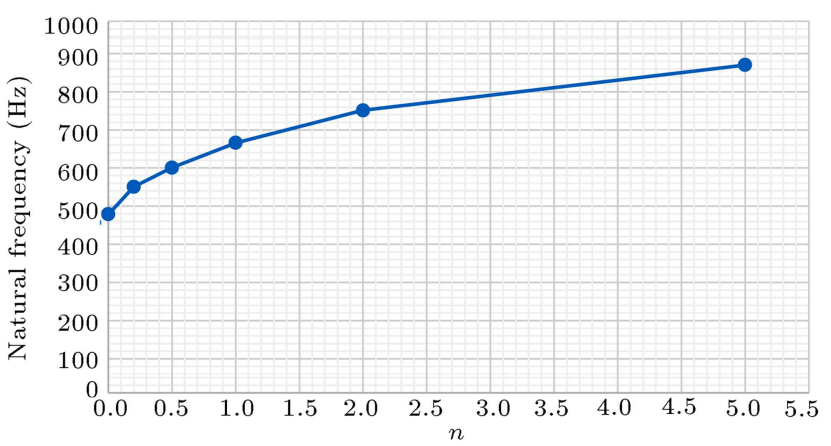

Figure 6. The first natural frequency for different values of $n$.

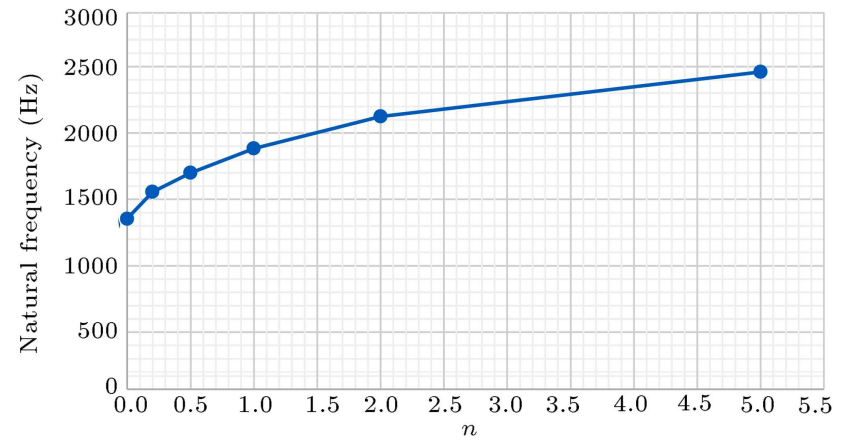

Figure 7. The second natural frequency for different values of $n$.

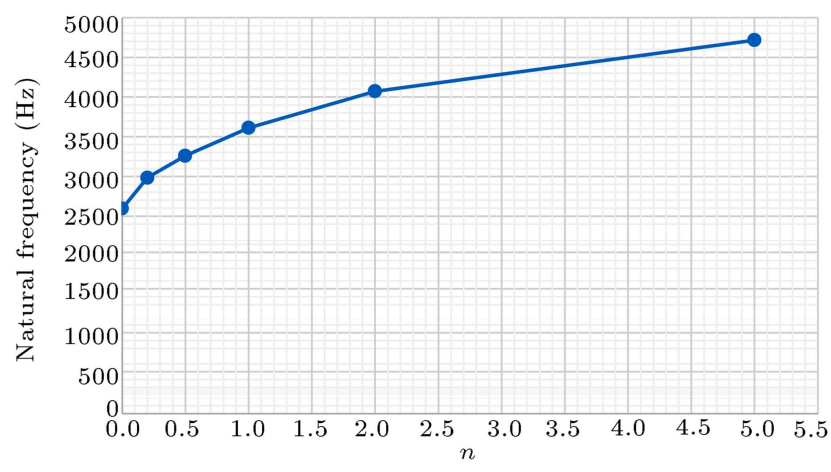

Figure 8. The third natural frequency for different values of $n$.

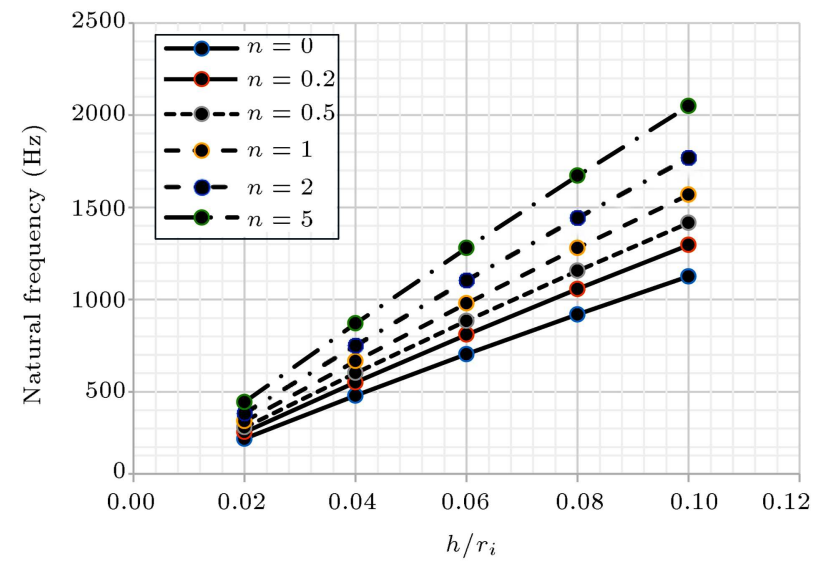

Figure 9. The first natural frequency for different values of $h / r_{i}$ and $n$.

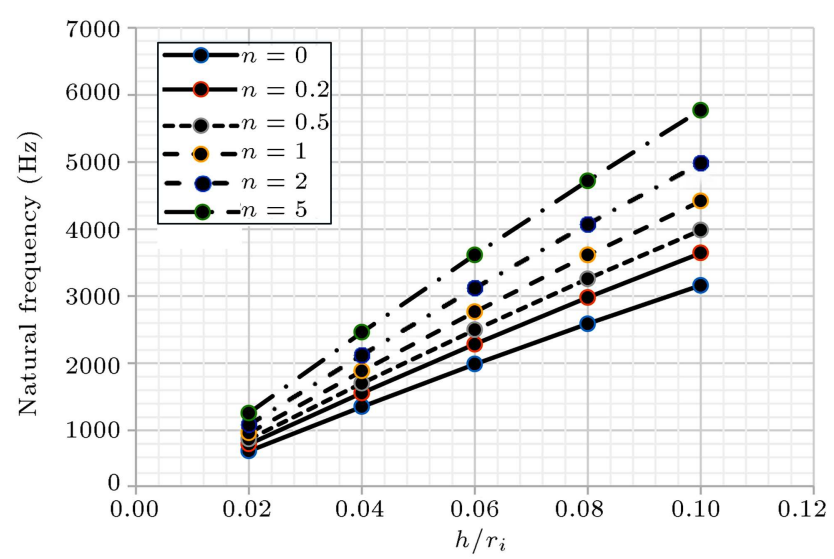

Figure 10. The second natural frequency for different values of $h / r_{i}$ and $n$. 


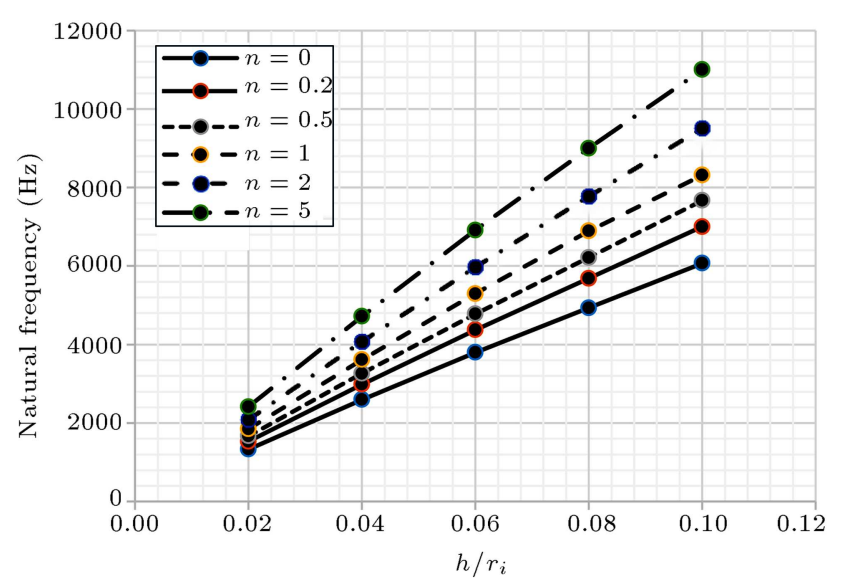

Figure 11. The third natural frequency for different values of $h / r_{i}$ and $n$.

performed. The power-law distribution is assumed in the thickness direction for FGM ring. Vibration analysis of rings is performed using superelement as well as conventional finite-element method. Findings indicate that the application of only six superelements to the rings brings about the same results as the conventional finite-element method with 10000 elements does. Saving in computational time using superelement could be nearly 0.01 , being much lower than that using conventional method. For simple uniform rings, the comparison of the obtained natural frequency using six superelements with analytical solution results in $0.21 \%$ error.

\section{References}

1. Rao, S., Vibration of Continuous Systems, John Wiley \& Sons, New Jersey (2007).

2. Wang, L., Lu, Y., Xiang, Y., Qin, L., and Cai, D. "Vibration analysis for piezoceramic ring", Ceramics International, 39(1), pp. 739-742 (2013).

3. Mustapha, K.B. "Modeling of a functionally graded micro-ring segment for the analysis of coupled extensional-flexural waves", Composite Structures, 117, pp. 274-287 (2014).

4. Loy, C.T., Larn, K.Y., and Reddy, J.N. "Vibration of functionally graded cylindrical shells", Int. J. Mech. Sci., 41(3), pp. 309-324 (1999).

5. Shakeri, M., Akhlaghi, M., and Hoseini, S.M. "Vibration and radial wave propagation velocity in functionally graded thick hollow cylinder", Composite Structures, 76(1-2), pp. 174-181 (2006).

6. Asgari, M. and Akhlaghi, M. "Natural frequency analysis of 2D-FGM thick hollow cylinder based on threedimensional elasticity equations", European Journal of Mechanics - A/Solids, 30(2), pp. 72-81 (2011).

7. Yin, S., Yu, T., and Liu, P. "Free vibration analysis of functionally graded plates using isogeometric finite element method", Journal of Vibration and Shock, 24, pp. 180-186 (2013).
8. Yu, T.T., Yin, S., Bui, T.Q., and Hirose, S. "A simple FSDT-based isogeometric analysis for geometrically nonlinear analysis of functionally graded plates", $F i$ nite Elem. Anal. Des., 96, pp. 1-10 (2015).

9. Sarvi, M.N. and Ahmadian, M.T. "Design and implementation of a new spherical super element in structural analysis", Applied Mathematics and Computation, 218(14), pp. 7546-7561 (2012).

10. Jiang, J. and Olson, M.D. "Nonlinear analysis of orthogonally stiffened cylindrical shells by a super element approach", Finite Elem. Anal. Des., 18(1-3), pp. 99-110 (1994).

11. Koko, T.S. and Olson, M.D. "Vibration analysis of stiffened plates by super elements", J. Sound Vib., 158(1), pp. 149-167 (1992).

12. Ju, F. and Choo, Y.S. "Superelement approach to cable passing through multiple pulleys", Int. J. Solids Struct., 42, pp. 3533-3547 (2005).

13. Ahmadian, M.T. and Bonakdar, M. "A new cylindrical element formulation and its application to structural analysis of laminated hollow cylinders", Finite Elem. Anal. Des., 44(9-10), pp. 617-630 (2008).

14. Taghvaeipour, A., Bonakdar, M., and Ahmadian, M.T. "Application of a new cylindrical element formulation in finite element structural analysis of FGM hollow cylinders", Finite Elem. Anal. Des., 50, pp. 1-7 (2012).

15. Ghorbani, Sh. and Ahmadian, M.T. "Extend the cylindrical superelement and its application to vibration analysis of cylindrical structures", Proceeding of the Fourth International Conference on Vibration and Acoustic, Tehran, Iran (2014) (in Persian).

16. Reddy, J.N., Theory and Analysis of Elastic Plates, Taylor and Francis, PA (1999).

17. Stasa, F.L., Applied Finite Element Analysis for Engineers, CBS, Japan (1986).

\section{Biographies}

Alireza Fatan obtained his MSc degree in Mechanical Engineering from Sharif University of Technology. He studied in the area of Dynamics and Vibrations and wrote his dissertation under the supervision of Prof. M.T. Ahmadian from 2013 to 2015. He also received his BSc degree in Mechanical Engineering from Shahre Kord University, ShahreKord, Iran, in 2013.

Mohammad Taghi Ahmadian obtained his PhD degree in Mechanical Engineering from University of Kansas in 1986. He served as an Assistant Professor in University of Missouri from 1984 to 1985, and did the same in University of Kansas from 1986 to 1987. He started working as an Assistant Professor in Sharif university of Technology from 1989. At present, he is a Professor in the School of Mechanical Engineering in Sharif University of Technology, Tehran, Iran. 